\title{
Origin and reduction of wakefields in photonic crystal accelerator cavities
}

\author{
Carl A. Bauer, ${ }^{1}$ Gregory R. Werner, ${ }^{1}$ and John R. Cary ${ }^{1,2}$ \\ ${ }^{1}$ Department of Physics, University of Colorado at Boulder, 390 UCB, Boulder, Colorado 80309, USA \\ ${ }^{2}$ Tech-X Corporation, 5621 Arapahoe Avenue, Boulder, Colorado 80303, USA
}

(Received 10 September 2013; published 13 May 2014)

\begin{abstract}
Photonic crystal $(\mathrm{PhC})$ defect cavities that support an accelerating mode tend to trap unwanted higherorder modes (HOMs) corresponding to zero-group-velocity $\mathrm{PhC}$ lattice modes at frequencies near the top of bandgaps. The effect is explained quite generally by photonic band and perturbation theoretical arguments. Transverse wakefields resulting from this effect are observed (via simulation) in a $12 \mathrm{GHz}$ hybrid dielectric $\mathrm{PhC}$ accelerating cavity based on a triangular lattice of sapphire rods. These wakefields are, on average, an order of magnitude higher than those in the $12 \mathrm{GHz}$ waveguide-damped Compact Linear Collider copper cavities. The avoidance of translational symmetry (and, thus, the bandgap concept) can dramatically improve HOM damping in PhC-based structures.
\end{abstract}

DOI: 10.1103/PhysRevSTAB.17.051301

PACS numbers: 42.70.Qs, 29.20.Ej, 41.60.-m, 84.40.-x

\section{INTRODUCTION}

Photonic crystals $(\mathrm{PhCs})$ have recently attracted interest from the accelerator community [1-12] for the following reasons: (1) $\mathrm{PhCs}$ enable the construction of accelerator cavities using dielectric materials. (2) PhC cavities intrinsically provide a wakefield damping mechanism. These features suggest an alternative to traditional (super)conducting cavity design, and therefore could result in higher gradients [13-17], lower power losses, and/or lower wakefields.

A simple argument from photonic band theory gives reason to believe that $\mathrm{PhC}$ cavities will have low wakefields. It says that, given a $\mathrm{PhC}$ with a band gap, a defect cavity will confine only one (ideally accelerating) mode to the defect; all other higher-frequency modes will propagate through the crystal and contribute minimally to the wakefields. This basic concept has led to the design of many defect cavity $\mathrm{PhC}$ acceleration schemes, a sample of which can be seen in the list of references above.

To date, only a handful of computational and experimental studies dedicated to wakefield damping in $\mathrm{PhC}$ cavities have been performed. In general, they have shown that the $Q$ factors of higher-order modes (HOMs) are much lower than that of the accelerating mode, indicating some wakefield damping [18-20]. These studies were performed on so-called hybrid $\mathrm{PhC}$ cavities (at $\mathrm{GHz}$ frequencies), where "hybrid" indicates the incorporation of both $\mathrm{PhC}$ and traditional (metal disc-loaded waveguide) design concepts (look ahead to Fig. 4 for an example of a hybrid $\mathrm{PhC}$ cavity) $[2,3,8]$.

Published by the American Physical Society under the terms of the Creative Commons Attribution 3.0 License. Further distribution of this work must maintain attribution to the author(s) and the published article's title, journal citation, and DOI.
This work looks in more detail at wakefield suppression in $\mathrm{PhC}$ cavities and reveals subtleties that can undermine the benefits implied by the simple band gap argument. These subtleties were uncovered in a thorough comparison between wakefield damping in a $12 \mathrm{GHz}$ hybrid $\mathrm{PhC}$ cavity based on a triangular lattice of sapphire rods (Fig. 4) and a $12 \mathrm{GHz}$ cavity from the main linac of the Compact Linear Collider, (CLIC) which uses side-coupled waveguides to damp HOMs (see Fig. 1) [21-24].

We find that, in the case of the hybrid $\mathrm{PhC}$ cavity (with sapphire rods) based on the triangular lattice, transverse wakefields are on average higher than those in the CLIC waveguide-damped cavity. A simple Fourier analysis of the transverse wake potential compared with the triangular lattice band diagram shows that the band edges (where the

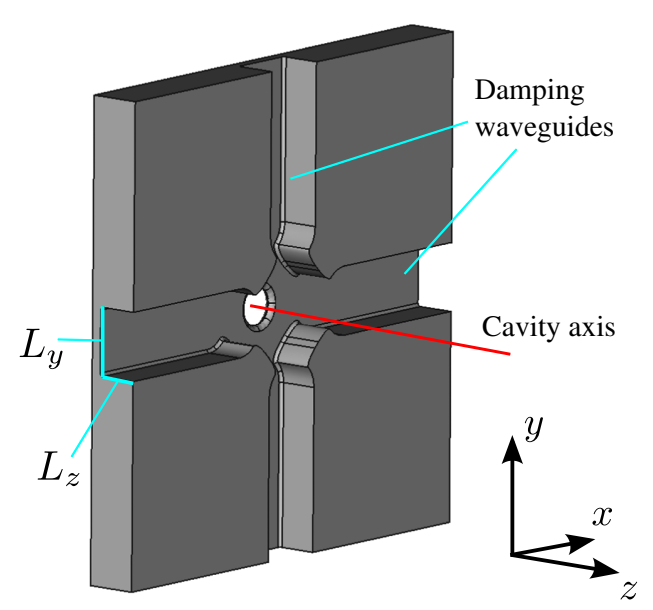

FIG. 1. In the above CLIC accelerating cell, [25] the four radial rectangular waveguides (terminated by electromagnetic absorbers) strongly damp HOMs; the cutoff frequency of each waveguide is slightly above the accelerating mode frequency and well below the lowest dipole frequency. 
dispersion curves flatten) are highly correlated with troublesome peaks in the transverse wake impedance. We attribute this correlation to the slow rate at which low-group-velocity $\mathrm{PhC}$ modes transport energy through the lattice.

The main result of this paper shows that the band gap property of PhC-based cavities does not necessarily predict strong wakefield suppression. In fact, for defect cavities, it may suggest the opposite. In a previous work, we discovered a 2D PhC-based cavity that strongly confines an accelerating mode without relying on the band gap principle [10]. In this paper, we simulate a 3D multicell adaptation of the 2D optimized cavity in Ref. [10] and compare wakefields with the CLIC cavity. While wakefields in the optimized (no-band-gap) cavity are lower than those in the lattice-based $\mathrm{PhC}$ cavity and similar to those in CLIC, we observe a significant reduction in the confinement of the accelerating mode, induced by the presence of the irises. Nevertheless, the result presents an interesting case in favor of nonlattice structures for wakefield suppression.

The following two sections review waveguide and $\mathrm{PhC}$ wakefield damping techniques. Section IV describes our simulation methods and results, comparing wakefields in the CLIC and lattice-based hybrid dielectric PhC cavities. Section V discusses the poor damping found in the triangular-lattice-based $\mathrm{PhC}$ cavity using photonic band theory. Finally, Sec. VI repeats the simulations of Sec. IV with an optimized cavity (from Ref. [10]) and compares these results with CLIC. Appendix A analyzes the performance of our numerical absorbers used in wakefield simulations. Appendix B discusses accelerating mode figures of merit (peak surface fields, accelerating efficiency, etc.) for each cavity type considered in this work.

\section{WAVEGUIDE DAMPING AND CLIC}

In the waveguide damping technique, wakefields are reduced by coupling HOMs out through waveguides terminated by electromagnetic absorbers. The CLIC design includes four radially directed waveguides in every cell, optimized to damp the $\mathrm{TM}_{110}$ cavity mode (since the $\mathrm{TM}_{110}$ mode, or lowest dipole mode, is the largest contributor to transverse wakefields). Figure 1 shows the second cell of the 26-cell constant-gradient CLIC cavity, TD26_vg1.8_R05_CC [24] (the second cell is the first "regular" cell, i.e., the first cell without power input couplers). The multicell cavity is formed by stacking pieces similar to that shown in Fig. 1.

The waveguides of the CLIC cavity effectively damp the lowest dipole mode (and other HOMs) without damping the accelerating mode. This desired behavior was obtained by carefully selecting the waveguide cross section such that the lowest waveguide cutoff frequency is between the accelerating mode and (undamped) dipole mode frequencies. For the CLIC cell in Fig. 1, the lowest waveguide cutoff frequency is $c / 2 L_{y}=13.6 \mathrm{GHz}\left(L_{y}=11 \mathrm{~mm}\right)$, the accelerating mode frequency is $12 \mathrm{GHz}$, and the frequency of an undamped dipole mode (i.e., dipole mode in a CLIC cell without damping waveguides) is approximately $21 \mathrm{GHz}$. Since the frequency of the accelerating mode is below waveguide cutoff, the mode remains confined to the cavity; the dipole mode, however, is free to propagate down the waveguides. The cutoff frequency is chosen far below the undamped dipole frequency because damping is less effective near cutoff where the group velocity (and thus speed of energy transport) of the waveguide mode vanishes $[26,27]$.

\section{INTRINSIC DAMPING IN PhCs}

Photonic crystals offer an alternative approach to wakefield damping: confine only the accelerating mode. This approach is made possible by the band gap property of some PhCs, and the resulting defect-mode phenomenon. A brief overview follows.

A band diagram succinctly summarizes the electromagnetic properties of PhCs. It is well known from Bloch theory that electromagnetic eigenmodes in periodic structures take the form

$$
\mathbf{E}(\mathbf{x}, t)=\tilde{\mathbf{E}}_{n}(\mathbf{x}) e^{i \mathbf{k} \cdot \mathbf{x}-i \omega_{n}(\mathbf{k}) t},
$$

where the reciprocal lattice vector $\mathbf{k}$ runs over a reduced region of $k$ space called the first Brillouin zone, $n$ is an integer indexing "bands" of solutions, and $\tilde{\mathbf{E}}_{n}$ has the periodicity of the lattice. A band diagram traditionally plots the resonant frequencies $\omega_{n}$ in each band along a representative $k$-space path within the first Brillouin zone. Figure 2(c) shows the TM band diagram for the $\mathrm{PhC}$ of interest to this paper [2D triangular lattice of sapphire discs-shown in Fig. 2(a)] along the $k$-space path shown in Fig. 2(b).

Of central importance in Fig. 2(c) is the wide range of frequencies between the first and second band in which there are no solutions. This feature is called a band gap, and is the basis for the formation of $\mathrm{PhC}$ resonant cavities that are of interest to the accelerator community. For a $\mathrm{PhC}$ with a band gap, a resonant cavity can often be formed by removing a single element from the lattice (creating a defect). This introduces a mode that oscillates at a frequency within the band gap and is necessarily localized to the defect. The defect TM mode for the sapphire triangular lattice is shown in Fig. 3 and oscillates at a frequency of $f=0.41 c / a$ - the center of the band gap; this mode can accelerate particles in/out of the page.

A single cell of a hybrid PhC cavity (Fig. 4) is formed from a $2 \mathrm{D} \mathrm{PhC}$ of sapphire rods (in this case a triangular lattice) sandwiched between two copper iris plates. As with the CLIC cavity, a multicell version would be assembled by stacking the element shown in Fig. 4. 
(a)

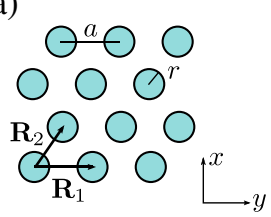

(c)

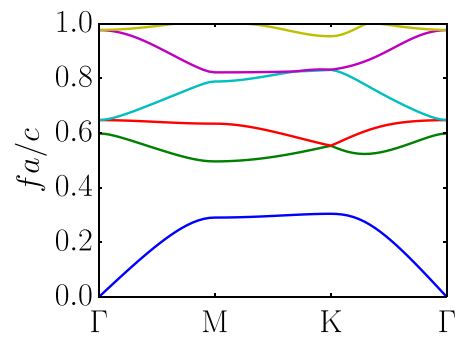

FIG. 2. Propagation in the 2D triangular lattice of sapphire discs (a) is forbidden for TM electromagnetic waves with frequencies near $0.4 c / a$ because of the band gap (c). Lattice vectors $\mathbf{R}_{1}$ and $\mathbf{R}_{2}$, interdisc spacing $a$, and disc radius $r$ are defined in (a). The first Brillouin zone of the reciprocal lattice is identified in (b) by the hexagon; the irreducible Brillouin zone is shaded and represents the entire Brillouin zone by symmetry. The dispersion curves for the lowest-frequency bands along the path outlining the shaded region in (b) are shown in (c) for $r=0.17 a$. Calculations were performed using the MIT Photonic Bands simulation code [28].

The dielectric constant of sapphire is anisotropic; in the case of Fig. 4, the $c$ axis of the sapphire is oriented along $z$. This means the dielectric tensor used in our calculations took the form $\varepsilon=9.4 \varepsilon_{0}(\hat{\mathbf{x}} \hat{\mathbf{x}}+\hat{\mathbf{y}} \hat{\mathbf{y}})+11.6 \varepsilon_{0} \hat{\mathbf{z}} \hat{\mathbf{z}}$ where $\varepsilon_{0}$ is the vacuum permittivity.

This structure intrinsically damps HOMs because frequencies above the band gap can propagate through the crystal (assuming no higher-frequency band gaps). However, as with waveguide damping, one must be wary

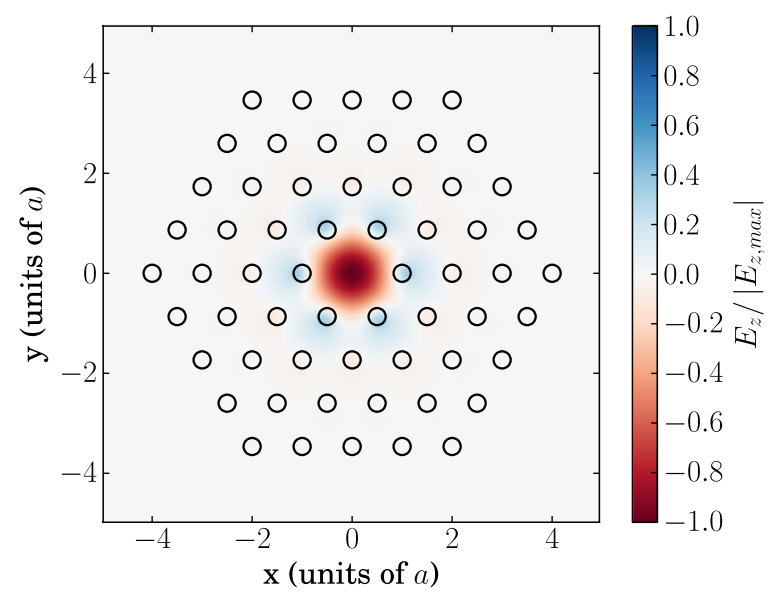

FIG. 3. Resonant TM defect cavity mode in a triangular lattice of lossless sapphire discs $(r=0.17 a)$. The lattice is truncated at 4 layers (60 discs), giving a radiative $Q$ factor of $Q_{\mathrm{rad}}=24,000$. $Q_{\text {rad }}$ increases exponentially with the number of layers.

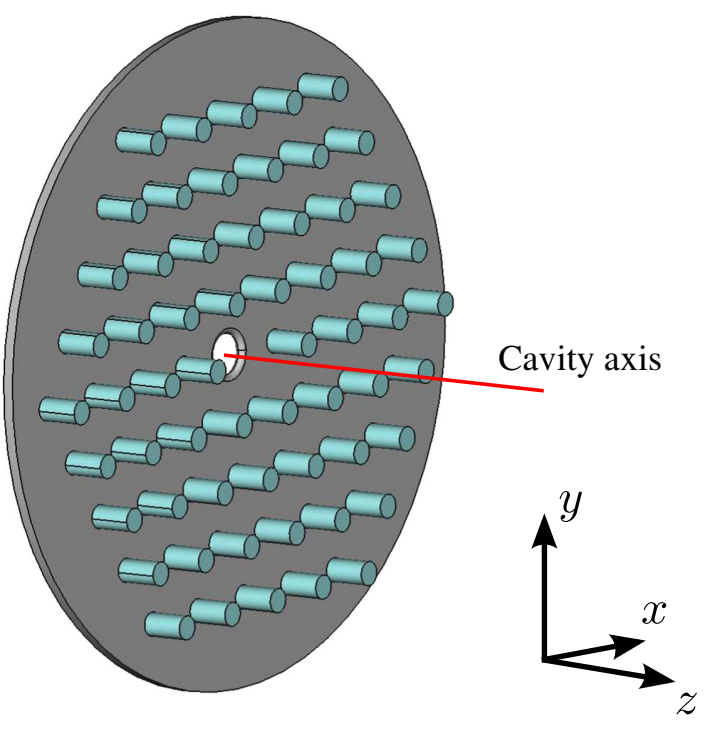

FIG. 4. The triangular lattice PhC of sapphire rods confines the accelerating mode to the cavity axis while damping HOMs.

of flat portions of the dispersion curves, where group velocity (and thus energy transport) vanishes [29]. In the following results and analysis, we find that for the 2D triangular lattice $\mathrm{PhC}$, dipole resonances in the defect are damped poorly by the surrounding $\mathrm{PhC}$. The cause is the coincidence of the dipole resonant frequency with the upper edge of the band gap, where the second band flattens.

\section{COMPARISON OF WAKEFIELD IN CLIC AND THE TRUNCATED PhC CAVITY}

We performed time-domain wakefield simulations for 8-cell versions of the CLIC (Fig. 1) and Tri-4-Sapphire (Fig. 4) cavities [30]. The 8 cells were identical in each cavity type (approximating the behavior of an infinitely periodic single cell). The iris geometry of the Tri-4Sapphire cavity was matched with the second cell of the TD26_vg1.8_R05_CC cavity, defined by the iris radius $a=3.15 \mathrm{~mm}$ and iris thickness $d=1.67 \mathrm{~mm}$; as a result, short-range wakefields (those wakefields affecting the drive bunch) are identical in both cavities.

Each cavity was driven by a highly relativistic electron bunch with a Gaussian profile in $z$, and a delta-function profile in $x$ and $y$. The Gaussian half-width of the bunch was $\sigma_{z}=1 \mathrm{~mm}$; therefore, HOMs up to frequencies of $\approx 90 \mathrm{GHz}$ were excited significantly. To excite transverse wakefields, the bunch was offset (from the cavity axis) by $1 \mathrm{~mm}$ in the $x$ direction. Wakefields were absorbed by normally conducting layers at the simulation domain boundaries (for further discussion on the performance of these absorbers, see Appendix A).

All simulations were implemented in the VORPAL framework which uses the finite-difference time-domain method for electromagnetics and the particle-in-cell technique for simulating the electron bunch and test particles [31]. In all 
simulations, grid cells were cubic with $\Delta z=0.3 \mathrm{~mm}$ so that the excitation bunch cutoff frequency was simulated with $\approx 10 \%$ error. Lower frequencies were therefore simulated with error $<10 \%$.

\section{A. Wake potential and impedance}

The wake potential is the net momentum change (normalized by charge) of a point charge trailing the wakefield excitation bunch. The longitudinal wake potential is defined as

$$
W_{z}\left(s, \mathbf{r}, \mathbf{r}^{\prime}\right)=-\frac{1}{q_{e}} \int_{0}^{L} E_{z}\left(z, \mathbf{r}, \mathbf{r}^{\prime}, t=(s+z) / v\right) d z,
$$

where $s$ is the point charge's distance (along $z$ ) behind the density peak of the Gaussian excitation bunch, $\mathbf{r}$ is the transverse position of the point charge (the cavity axis is the transverse origin), $\mathbf{r}^{\prime}$ is the transverse position of the excitation bunch, $v$ is the bunch/point charge velocity (basically $c$ ), $L$ is the total length of the multicell cavity, and $q_{e}$ is the excitation bunch charge. Similarly, the transverse wake potential is defined as

$\mathbf{W}_{\perp}\left(s, \mathbf{r}, \mathbf{r}^{\prime}\right)=\frac{1}{q_{e}} \int\left(\mathbf{E}_{\perp}+v \hat{z} \times \mathbf{B}_{\perp}\right)\left(z, \mathbf{r}, \mathbf{r}^{\prime}, t=(s+z) / v\right) d z$.

In the theoretical case of a cylindrically symmetric cavity with infinitely long beam tubes, the form of the $m$ th-order azimuthal multipole of the wake potential (for $|\mathbf{r}|<a$ ) is particularly simple [32]:

$$
\begin{aligned}
W_{z, m}\left(r, r^{\prime}, \phi, s\right)= & X_{m}(s) r^{m} r^{\prime m} \cos m \phi, \\
\mathbf{W}_{\perp, m}\left(r, r^{\prime}, \phi, s\right)= & m Y_{m}(s) r^{m-1} r^{\prime m} \\
& \times(\cos m \phi \hat{r}-\sin m \phi \hat{\phi}),
\end{aligned}
$$

where $X_{m}(s)$ and $Y_{m}(s)$ become the quantities of interest, independent of transverse coordinates [30,33]. The value of $\phi^{\prime}$ has been set to zero in the above since only the difference in azimuth $\left(\phi-\phi^{\prime}\right)$ appears in the above expressions. The transverse wake potential [Eq. (5)] is zero when $m=0$; only modes with $m>0$ mediate transverse kicks [32].

The longitudinal and transverse wake potentials are dominated by monopole and dipole wakefields, respectively. The expressions for these contributions are simpler still:

$$
\begin{gathered}
W_{z, 0}=X_{0}(s), \\
\mathbf{W}_{\perp, 1}=\mathbf{r}^{\prime} Y_{1}(s) .
\end{gathered}
$$

Both expressions are uniform throughout the beam tube region (independent of $r$ and $\phi$ ) and the dipole transverse wake potential is proportional to and in the direction of the drive beam's transverse offset.

The structures under consideration are not cylindrically symmetric; nevertheless, the above forms are assumed in our calculations since the forms hold well for wake potentials near the cavity axis [18,32]. Our numerical calculations produce $W_{z}\left(\mathbf{r}, \mathbf{r}^{\prime}, s\right)$ and $\mathbf{W}_{\perp}\left(\mathbf{r}, \mathbf{r}^{\prime}, s\right)$; we plot the normalized wake potentials, $X_{m}(s)$ and $Y_{m}(s)$, by extracting multipole contributions and dividing by the radial offsets of the drive bunch and wakefield sample points (details in next paragraph). We are mainly interested in the monopole and dipole contributions (i.e., the largest longitudinal and transverse kicks) per unit length of the multicell cavity, and therefore plot $X_{0}(s) / L$ and $X_{1}(s) / L$, in units of $\mathrm{V} /[\mathrm{pCm}]$ and $\mathrm{V} /[\mathrm{pCmmm}]$, respectively.

The wake potential was calculated by chasing the drive electron bunch with test particles of very low charge (so that wakefields were not induced by the test particles), but equally diminished mass to retain the electron charge-mass ratio. The net momentum change of the test particles gives the wake potential after division by the excitation bunch charge and test particle charge. The test particles were organized in rings so that the multipole contributions to the wake potential could be extracted. One ring of 6 test particles was emitted from the $-z$ domain boundary at every time step following emission of the drive bunch. The $n$th test particle in each ring was given the transverse position:

$$
\begin{aligned}
& r=1 \mathrm{~mm}, \\
& \phi=\frac{2 \pi n}{6} .
\end{aligned}
$$

The wake impedance is simply the Fourier transform of the wake potential. Under the approximation of cylindrical symmetry, we focus on the core multipole contributions to the wake impedance, $\tilde{X}_{m}(f)$ and $\tilde{Y}_{m}(f)$, defined by

$$
\begin{aligned}
& \tilde{X}_{m}(f)=\frac{1}{c} \int_{0}^{\infty} X(s) e^{-i 2 \pi f s / c} d s, \\
& \tilde{Y}_{m}(f)=\frac{1}{c} \int_{0}^{\infty} Y(s) e^{-i 2 \pi f s / c} d s,
\end{aligned}
$$

where $f$ is frequency. The above relations are approximated by the discrete Fourier transform in our time-domain simulations. The monopole-longitudinal and dipoletransverse wake impedances are $\tilde{X}_{0}(f)$ and $\tilde{Y}_{1}(f)$, respectively.

\section{B. Results}

The envelopes (lines connecting local maxima/minima) of the longitudinal monopole wake potentials are shown in Fig. 5 with the corresponding wake impedances shown in Fig. 6. Fluctuations of the envelope give a qualitative 


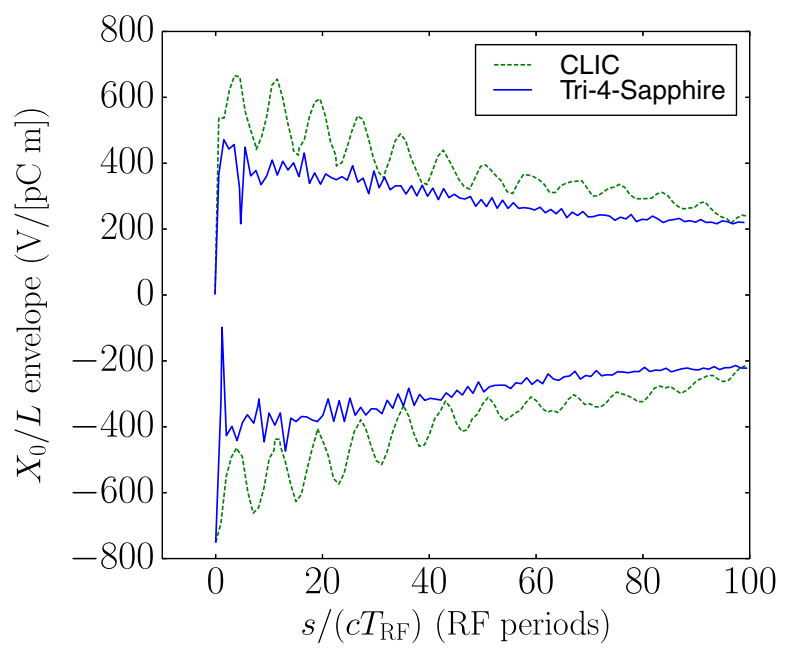

FIG. 5. Oscillations in the envelope of the monopole wake potential indicate the presence of monopole HOMs. The rf period is $T_{\mathrm{rf}}=(12 \mathrm{GHz})^{-1}=83.3 \mathrm{ps}$.

picture of the presence of HOMs (showing the full wake potential only serves to confuse the plot). The impedances in Fig. 6 show lower peaks for the Tri-4-Sapphire cavity, indicating stronger monopole HOM damping and/or weaker monopole HOM excitation. The mode density is significantly higher in Tri-4-Sapphire.

Amplitudes of the transverse dipole wake potentials are shown in Fig. 7 with the corresponding impedances shown in Fig. 8. As a reference, we have included in each plot the transverse dipole wake potentials in the empty cavitydefined most easily as an 8-cell hybrid $\mathrm{PhC}$ cavity without any rods. The empty cavity represents ideal wakefield damping but does not confine an accelerating mode. Dipole wakes are relatively high in the Tri-4-Sapphire cavity; the frequencies of the troublesome HOMs are indicated by the sharp peaks around $15 \mathrm{GHz}$ in Fig. 8. This result motivates

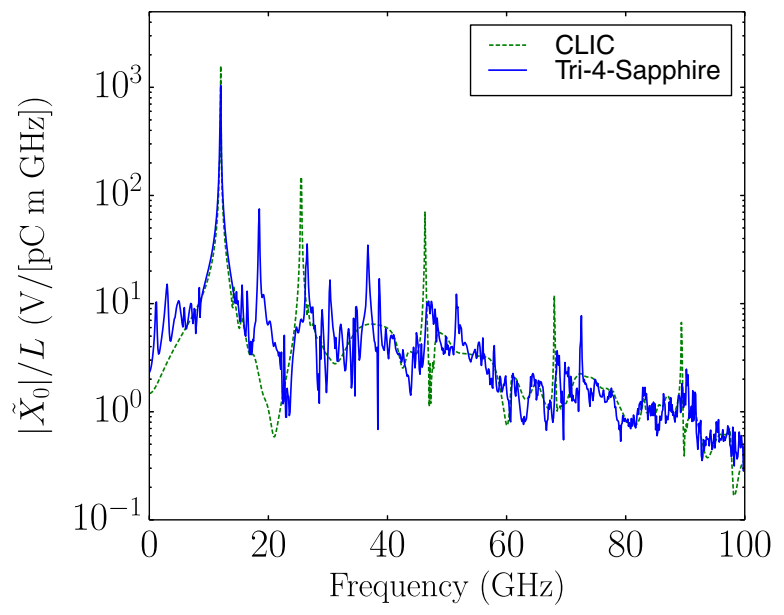

FIG. 6. Monopole HOMs are relatively numerous in the Tri-4Sapphire cavity, but occur at lower amplitudes.

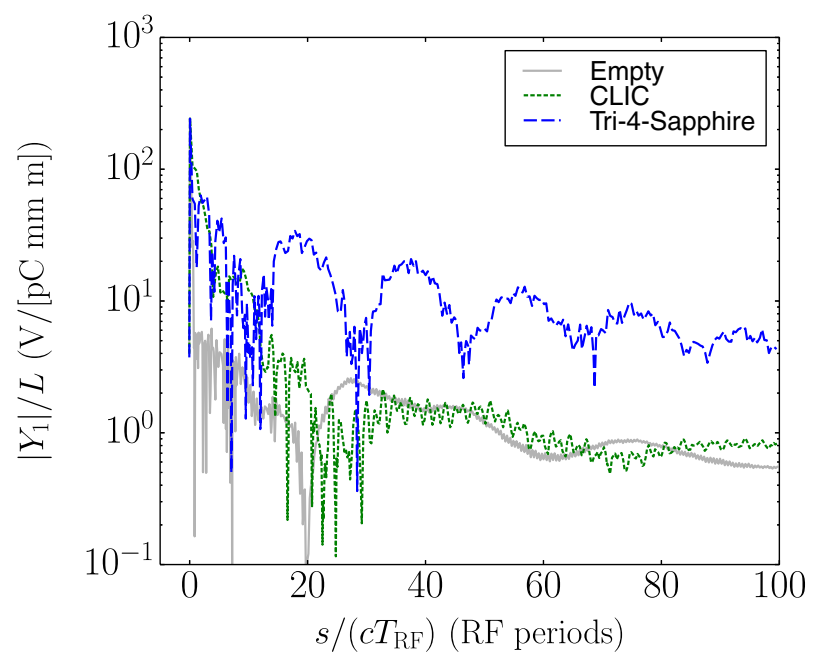

FIG. 7. Transverse dipole wake potentials in the Tri-4Sapphire cavity are (on average) an order of magnitude higher than those in CLIC.

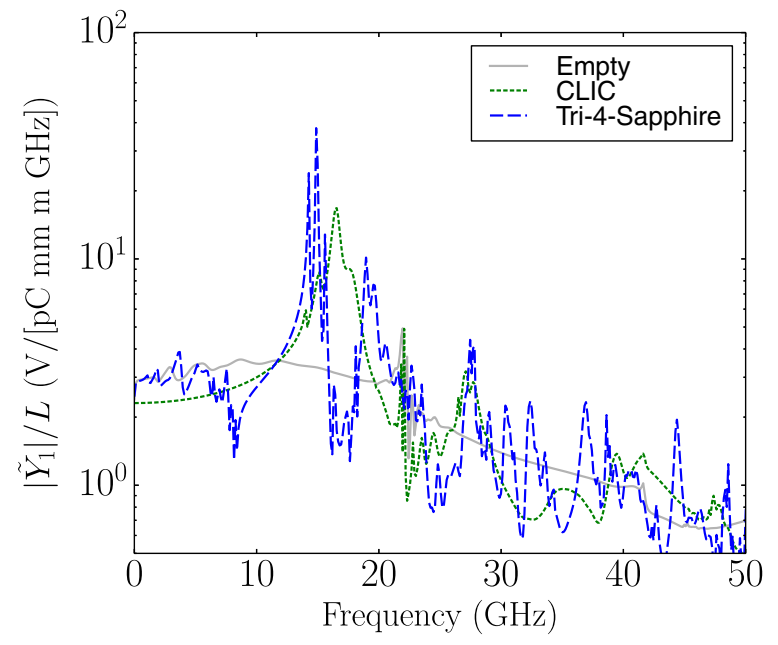

FIG. 8. The higher transverse wake in the Tri-4-Sapphire cavity is due to the cluster of "high"- $Q$ modes around $15 \mathrm{GHz}$.

a more careful analysis of the intrinsic PhC damping mechanism and prompts the following discussion.

\section{ORIGIN OF WAKEFIELDS IN THE TRUNCATED PhC CAVITY}

The transverse dipole impedance in the Tri-4-Sapphire cavity shows that the dipole wake potential mostly comprises a cluster of modes near $15 \mathrm{GHz}$. In this section, we explain the presence of these modes using the properties of the triangular-lattice band structure: flat regions of the dispersion diagram imply low-group-velocity $\mathrm{PhC}$ modes which sluggishly transport energy and thus cannot effectively damp cavity modes to which they are strongly coupled. This issue was addressed briefly in Ref. [29] 
within a study of a square-lattice-based metal-rod accelerating structure.

We have discussed how waveguide damping is effective for coupling out frequencies only above cutoff (the further above cutoff the better). This is because waveguide modes with frequencies near cutoff have vanishing group velocities (because of the flattened dispersion at cutoff) and thus propagate slowly down the waveguide- effectively, they are trapped. In $\mathrm{PhCs}$, the dispersion tends to flatten where the spatial variation of the modes matches the spatial variation of the dielectric, introducing $\mathrm{PhC}$ modes with vanishing group velocities.

Figure 9 shows the transverse dipole impedance of the Tri-4-Sapphire cavity in the frequency range $0-25 \mathrm{GHz}$ (a zoom view of Fig. 8) and matches it with the 2D TM band diagram for the triangular lattice of sapphire discs. The two most prominent peaks in the impedance clearly line up with the flat portions of the second band (the third peak can be matched with another flat band in the fully 3D band diagram). The annotated impedance peak (second largest) was investigated further using the time-domain mode extraction technique. Figure 10 shows the field pattern of the extracted mode (from a periodic single-cell simulation at phase advance $\phi \approx 3 \pi / 4$ ) and compares it with the 2D PhC lattice mode at the $M$ point of the second band. The field patterns clearly match.

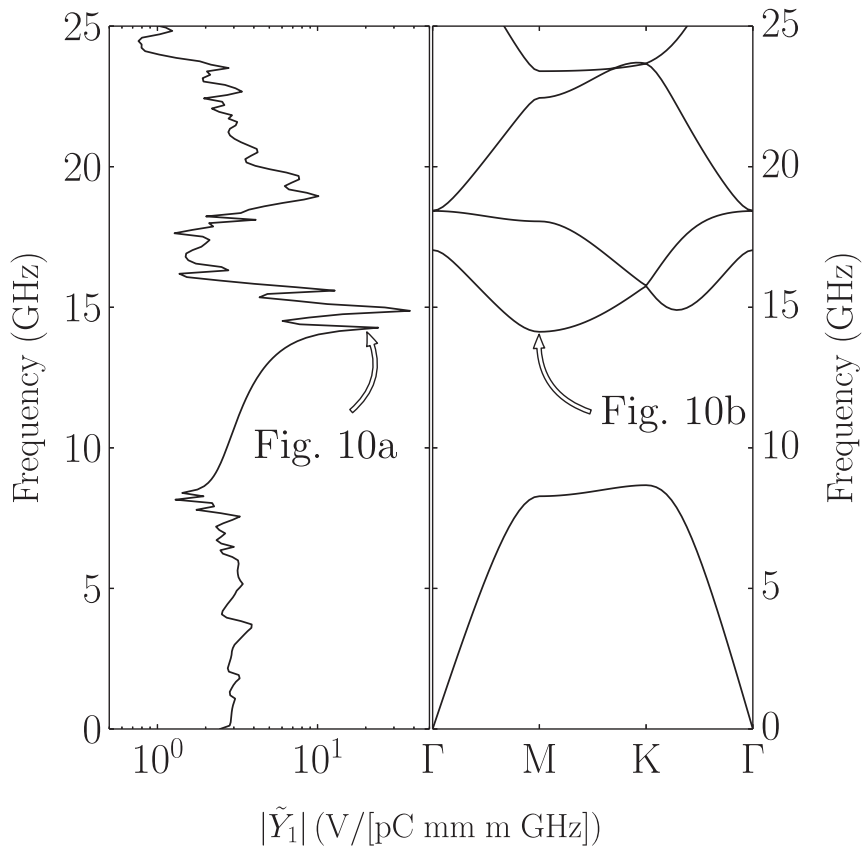

FIG. 9. The left plot shows a zoomed-in view of the troublesome part of the transverse dipole impedance (from Fig. 8). The right plot is the 2D TM band diagram for the triangular lattice of sapphire discs. The annotated peak in the impedance is at the same frequency as the $M$ point of the second band (also see the mode patterns in Fig. 10). This correlation supports the idea that low- $v_{g}$ modes pose a problem for wakefields.
Both the damping waveguides and the triangular $\mathrm{PhC}$ suffer from low-group-velocity modes. Why then is the damping worse in the Tri-4-Sapphire cavity? The answer lies in the combined characteristics of the damping structure and the central cavity region. Consider the lowest dipole mode in the CLIC cavity. Section II showed that waveguide damping is more effective when the undamped resonant frequency is further above the cutoff frequency. The central cavity dimensions in the CLIC cavity determine a dipole resonant frequency; thus, the waveguide

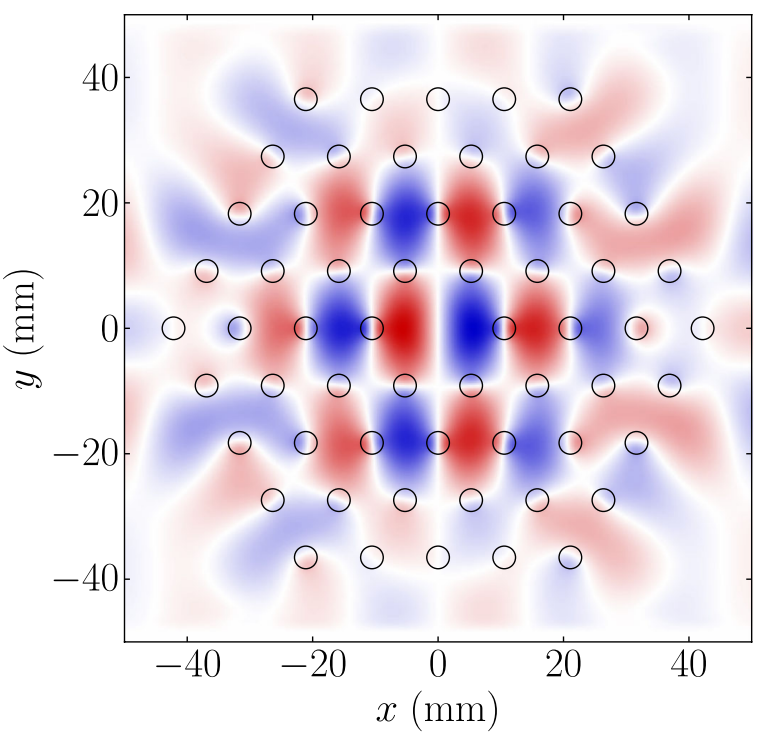

(a) Tri-4-Sapphire mode

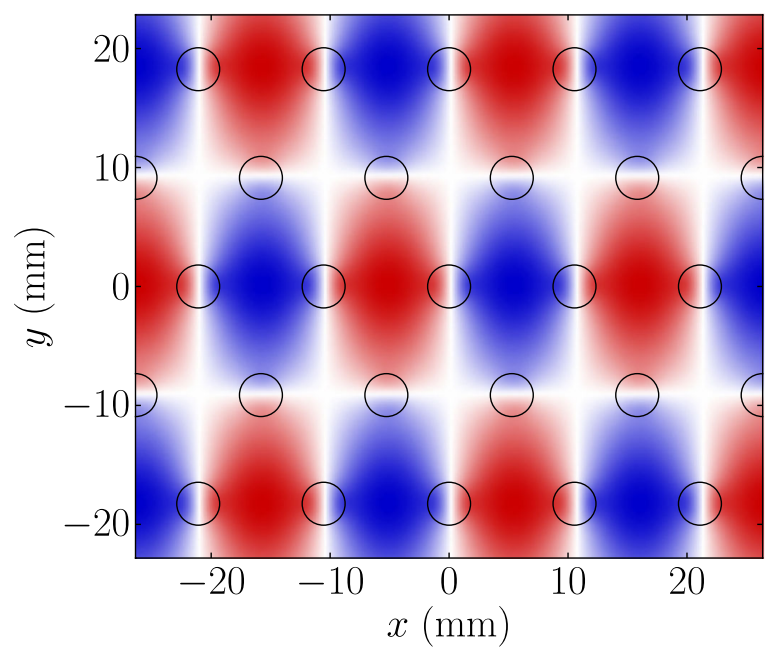

(b) Infinite $\mathrm{PhC}$ mode

FIG. 10. Analagous modes in (a) the Tri-4-Sapphire periodic single-cell cavity and (b) an infinite $2 \mathrm{D} \mathrm{PhC}$ [the mode pattern in (b) is uniform in $z$ ]. The propagating $\mathrm{PhC}$ mode in (b) has a vanishing group velocity; thus, its counterpart in (a) is "trapped" and contributes significantly to the dipole wakefield. Computations used (a) VORPAL [31] and (b) MIT Photonic Bands [28]. 
dimensions are selected such that cutoff is as far below that frequency as possible (without affecting the accelerating mode too much). The gap between the accelerating frequency and the dipole frequency is large enough such that this scheme results in effective dipole damping.

In contrast, the defect region of the Tri-4-Sapphire cavity is (by definition) highly commensurate with the geometry of the surrounding lattice. Furthermore, the mode patterns in Fig. 10 show little electric field energy inside the dielectric, indicating that the removal of the central rod has a small, perturbative effect (compared to modes at the top of the first band, which have most of their field energy concentrated in dielectric). (For an introduction to electromagnetic perturbation theory, see Ref. [34].) As a result, the dipolar defect resonance is at the same frequency as the low-group-velocity lattice mode (or the "cutoff" mode), and is thus poorly damped. Put another way, the creation of the defect weakly perturbs the band-edge lattice mode; therefore, the mode retains a strong presence in the defect. This argument suggests pushing the inner layer of rods closer to the cavity axis, which is likely to increase the dipole resonance frequency in the defect above the flat portion of the lattice band and thus increase coupling to higher-group-velocity lattice modes.

The situation may be exacerbated by the impedance mismatch at the outer layer of rods. Because of its lowgroup-velocity, the lattice mode shown in Fig. 10 may be highly susceptible to reflections off of the transition between lattice and vacuum, effectively increasing its confinement. This transition could be made smoother by slowly decreasing the radii of the rods in outer layers but would add significantly to the transverse size of the structure.

\section{WAKEFIELDS IN OPTIMIZED HYBRID PhC CAVITIES}

In a previous work, 2D simulations showed that some rod arrangements lacking any lattice symmetry (but retaining some rotational symmetry) dramatically reduce the radiative losses of the accelerating mode as compared to lattice arrangements of equal rod count [10]. Subsequent 3D simulations indicated lower wakefields in these optimized structures [18]; however, the simulations in that study were of single-cell closed cavities (no irises or beam tubes) and made comparisons with only the pillbox. In this section, we investigate the wakefield-damping performance of a $12 \mathrm{GHz}$ multicell optimized PhC cavity with irises and compare with the CLIC cavity.

A single cell of the multicell optimized cavity that we simulate (henceforth referred to as Opt-18-Sapphire-rods are sapphire) is pictured in Fig. 11. The transverse positions and radii of the rods were calculated from their $2 \mathrm{D}$ values in Ref. [10] using a uniform transverse coordinate scaling (relative to the cavity axis) that resulted in an accelerating mode frequency of $12 \mathrm{GHz}$ for the desired iris dimensions.

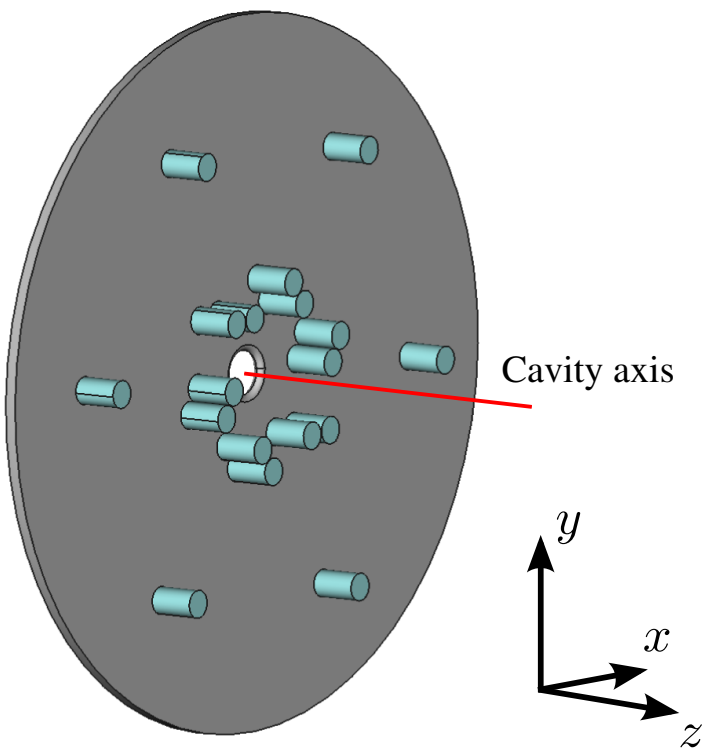

FIG. 11. An optimized hybrid PhC cavity (Opt-18-Sapphire) reduces the number of rods required to confine the accelerating mode.

Irises perturb the frequency, $Q$ factor, and field pattern of the accelerating mode away from their closed-cavity (essentially 2D) values. The $Q$ factor of metal cavities and $\mathrm{PhC}$ cavities with a band gap is robust to slight perturbations; for example, a perturbation of the frequency in a $\mathrm{PhC}$ cavity should not affect the $Q$ factor too much if the frequency remains in the band gap. In contrast, Ref. [10] showed that the $Q$ factor of an optimized cavity can be quite sensitive to perturbations. Indeed, we find that the irises in the multicell cavity of this paper significantly reduce the $Q$ factor below its 2D value (by roughly $85 \%$ ).

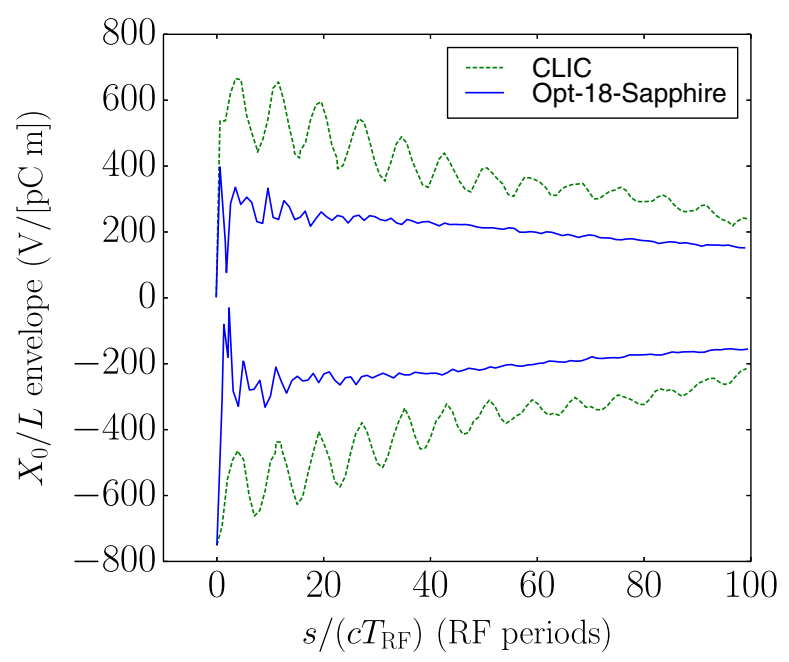

FIG. 12. The monopole wake potential envelope is smoother for the Opt-18-Sapphire cavity. However, the amplitude of the accelerating mode is lower, indicating weaker coupling to the beam (see Appendix B). 


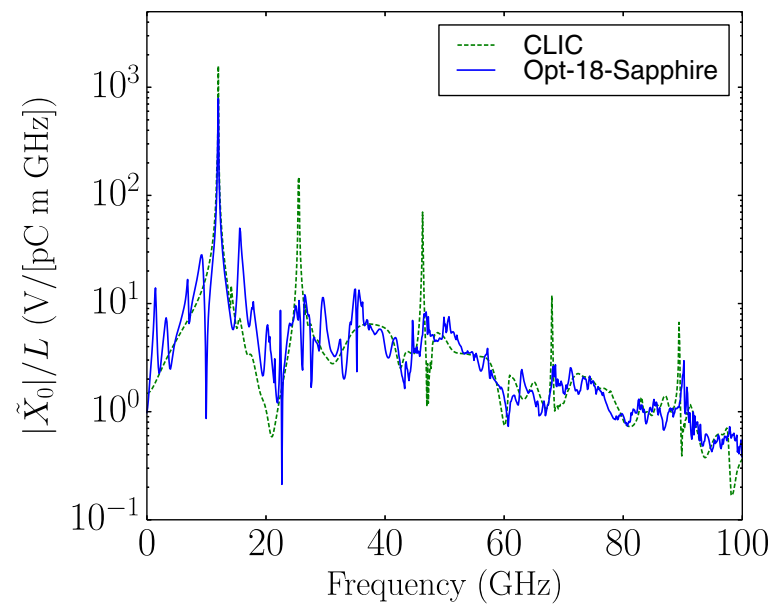

FIG. 13. Monopole HOMs are numerous but suppressed in the Opt-18-Sapphire cavity.

It is possible that a corrective perturbation to the rod positions would return the $Q$ factor to its 2D value. However, such an optimization in 3D would require significant additional computational effort. Without a clear connection between the $Q$ factor of the accelerating mode and the damping of higher-order modes in the optimized cavity, we compare wakefields in Opt-18-Sapphire with wakefields in CLIC as a preliminary study of damping in nonlattice-based (optimized) hybrid $\mathrm{PhC}$ cavities.

Figures 12-15 detail the wake potentials of an 8-cell Opt-18-Sapphire cavity and compare with CLIC. Monopole HOMs are more numerous but less prominent in the Opt-18-Sapphire cavity. Transverse wakes in Opt-18Sapphire are similar to CLIC on average but greatly reduced in comparison with Tri-4-Sapphire (cf. Fig. 7). Unfortunately, the analysis of Sec. V cannot be applied to Opt-18-Sapphire because of the lack of any band structure; however, these results suggest that nonlattice structures

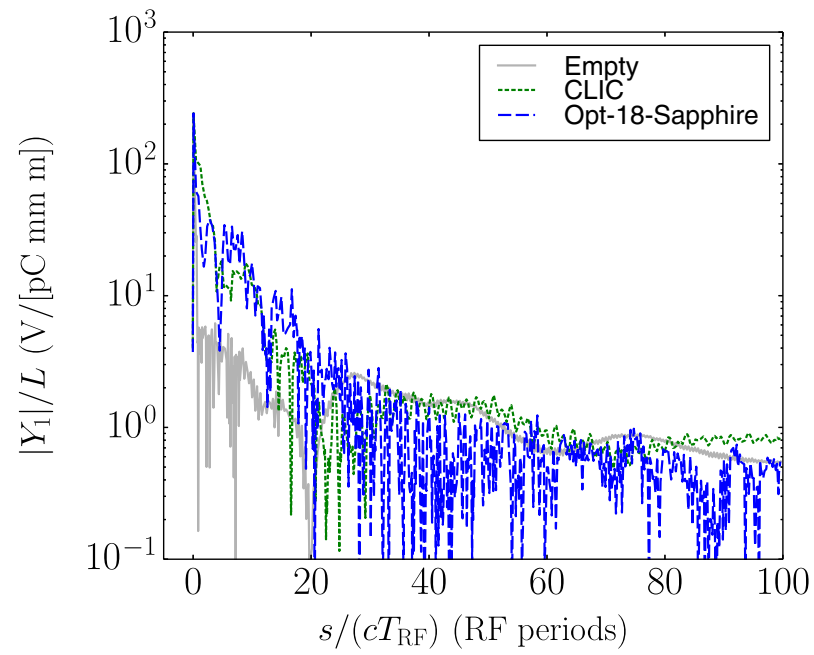

FIG. 14. Transverse dipole wake potentials in 8-cell cavities using conducting absorbers.

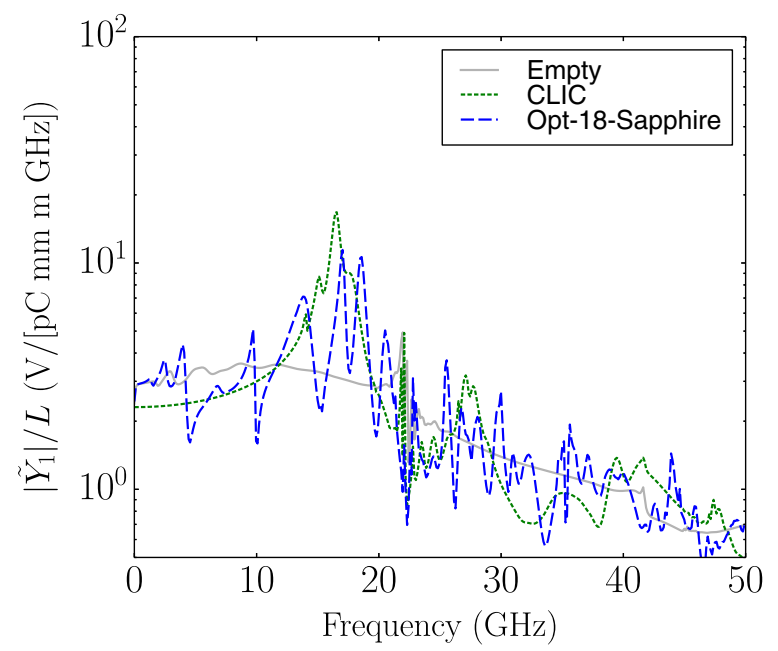

FIG. 15. Transverse dipole impedance in 8-cell cavities using conducting absorbers.

could avoid the inherently poor damping characteristics found in PhC defect cavities.

\section{CONCLUDING REMARKS}

Low wakefields require that the energy in HOMs be dissipated as quickly as possible. Therefore, a damping mechanism (or structure) should have a mode spectrum without any low group velocities. In principle, the CLIC cavity suffers from low- $v_{g}$ HOMs near the cutoff frequency of its damping waveguides; however, the sparse mode density of the conducting cavity allowed the placement of the cutoff frequency within an empty region of the spectrum, producing effective damping. $\mathrm{PhC}$ cavities suffer from low- $v_{g}$-confined HOMs due to the flattening of bands near $k$ points of strong lattice symmetry. Specifically, in the Tri-4-Sapphire design, the defect-based cavity supports dipolar resonances that strongly couple to band-edge $\mathrm{PhC}$ modes with low $v_{g}$.

Future work should focus on reducing wakefields in lattice-based $\mathrm{PhC}$ cavities because the lattice structure has the potential to reduce surface magnetic fields and increase accelerating efficiencies (see Appendix B) and $\mathrm{PhC}$ band theory provides an explanation for the existence of troublesome HOMs. Possible routes to reducing wakes in Tri-4Sapphire cavities include perturbing the central defect region to eliminate low- $v_{g}$ resonances and/or reducing impedance mismatch at the truncation of the lattice. Given the low wakefields in the Opt-18-Sapphire cavity (and its high $Q_{\text {rad }}$ to rod-count ratio), brute-force optimization of rod positions to lower wakefields is also recommended.

\section{ACKNOWLEDGMENTS}

This work was supported by the U.S. Department of Energy Grant No. DE-FG02-04ER41317. Most of the simulations described in this work were performed with 
the VORPAL framework; we would like to acknowledge the efforts of the Vorpal team: D. Alexander, K. Amyx, E. Angle, T. Austin, G. I. Bell, D. L. Bruhwiler, E. CormierMichel, Y. Choi, B. M. Cowan, R. K. Crockett D. A. Dimitrov, M. Durant, B. Jamroz, M. Koch, S. E. Kruger, A. Likhanskii, M.C. Lin, M. Loh, J. Loverich, S. Mahalingam, P. J. Mullowney, C. Nieter, K. Paul, I. Pogorelov, C. Roark, B. T. Schwartz, S. W. Sides, D. N. Smithe, P. H. Stoltz, S. A. Veitzer, D. J. Wade-Stein, N. Xiang, C. D. Zhou. We would also like to thank Alexej Grudiev at CERN for providing the CLIC cavity geometry.

\section{APPENDIX A: ABSORBERS}

To absorb wakefields, the ends of each damping waveguide and the transverse edges of the $\mathrm{PhC}$ simulations were filled with blocks of conducting material with a special conductivity profile aimed at minimizing reflections off of the cavity-facing surface. The profile is given by

$$
\sigma(w)=\sigma_{\max }\left(\frac{w}{W}\right)^{2},
$$

where $w$ is depth in the conductor and $W$ is the entire depth of the conducting block. The quadratic form resembles damping techniques used in practice [35], where a cone of absorbing material is placed at the end of a damping waveguide. Our calculations used $W=25 \mathrm{~mm}$ and $\sigma_{\max } / \varepsilon_{0}=4.7 \omega_{\text {acc }}$ where $\omega_{\text {acc }}=2 \pi 12 \mathrm{GHz}$.

We have found that the additional contribution to the wake potentials due to reflections off of the conducting absorbers are higher in the CLIC cavity than in the hybrid $\mathrm{PhC}$ cavities. These findings were obtained from simulations with large transverse extents $-125 \mathrm{~mm}$ from cavity axis to simulation edge in both $x$ and $y$ (simulations in Sec. IV had approximately $40 \mathrm{~mm}$ between the cavity axis and the start of the absorbers). Wakefield reflections off of the absorbers at $40 \mathrm{~mm}$ show up in the wake potential at $s \gtrsim 80 \mathrm{~mm}$, whereas reflections from $125 \mathrm{~mm}$ simulation edges show up at $s \gtrsim 250 \mathrm{~mm}$. Transverse dipole wake potential results were compared at distances of $80 \mathrm{~mm}<$ $s<250 \mathrm{~mm}$ (see Fig. 16). Differences in this region were significant for the CLIC cavity and minor in all hybrid $\mathrm{PhC}$ cavities. This means wakefield damping can be improved by reducing the impedance mismatch of the absorber in the CLIC waveguides, whereas improving absorption in the $\mathrm{PhC}$ cavities will have little impact on the wake potentials.

\section{APPENDIX B: ACCELERATING MODE COMPARISON}

Accelerator cavity design is a complex multidimensional optimization process that tries (in no particular order) (1) to maximize the accelerating electric field (or gradient) so that high energies are reached over short distances, (2) to

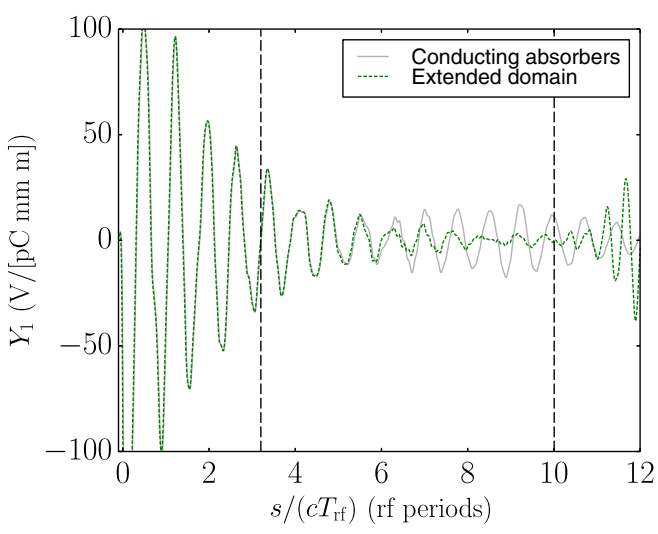

(a) CLIC

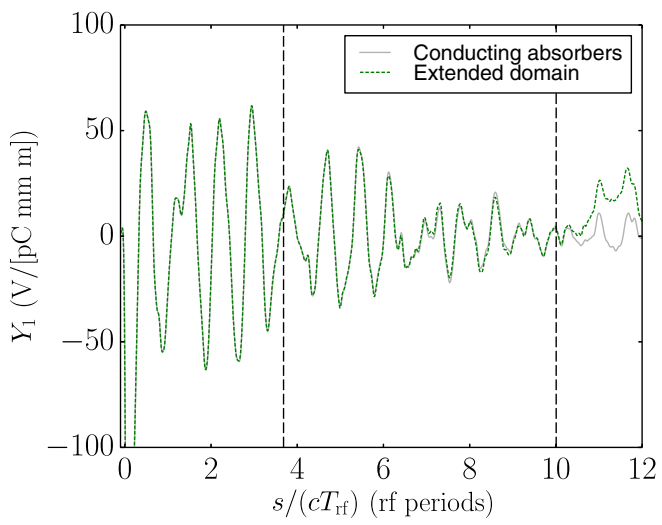

(b) Tri-4-Sapphire

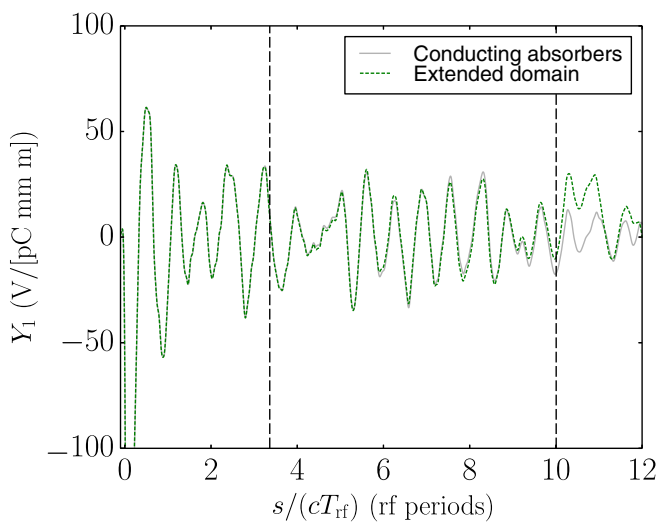

(c) Opt-18-Sapphire

FIG. 16. Reflections off of the conducting absorbers cause differences in the wake potentials between the two vertical lines (more so in the CLIC cavity). Beyond the second line, reflections from the extended domain boundaries reach the cavity axis.

maximize the transfer of electromagnetic power to the beam, and (3) to minimize wakefields to avoid beam instabilities and breakup. The main body of this paper focused on wakefields in hybrid $\mathrm{PhC}$ cavities [i.e., goal (3)]. This appendix addresses some aspects of goals (1) and (2) so that a more complete comparison between the CLIC and hybrid $\mathrm{PhC}$ cavities may be drawn. 
TABLE I. Figures of merit for the accelerating mode in periodic single cell cavities. $a=3.15 \mathrm{~mm}, d=1.67 \mathrm{~mm}, \phi=2 \pi / 3$. All simulations performed at $\Delta z / d=8$.

\begin{tabular}{lcccc}
\hline \hline & Pillbox & CLIC & $\begin{array}{c}\text { Tri-4- } \\
\text { Sapphire }\end{array}$ & $\begin{array}{c}\text { Opt-18- } \\
\text { Sapphire }\end{array}$ \\
\hline$v_{g} / c(\%)$ & 1.83 & 1.65 & 1.16 & 0.78 \\
$Q_{\text {metal }}$ & 6,700 & 5,900 & 11,400 & 11,400 \\
$Q_{\text {rad }}$ & $\infty$ & $\infty$ & 26,600 & 3,800 \\
$Q_{\text {diel }}$ & $\infty$ & $\infty$ & 67,000 & 39,000 \\
$Q_{\text {total }}(\mathrm{M} \Omega / \mathrm{m})$ & 6,700 & 5,900 & 7,100 & 2,700 \\
$r_{\text {shunt }}$ & 106 & 82 & 70 & 18 \\
$k(\mathrm{~V} / \mathrm{pC} / \mathrm{m})$ & 298 & 260 & 187 & 125 \\
$E_{\text {surf,metal,max }} / E_{\text {acc }}$ & 1.93 & 1.96 & 1.93 & 1.93 \\
$c B_{\text {surf,metal,max }} / E_{\text {acc }}$ & 1.0 & 1.54 & 1.49 & 1.73 \\
$E_{\text {surf,diel,max }} / E_{\text {acc }}$ & $\ldots$ & $\ldots$ & 0.54 & 0.64 \\
$c B_{\text {surf,diel,max }} / E_{\text {acc }}$ & $\ldots$ & $\ldots$ & 1.26 & 1.79 \\
$E_{\text {diel, max }} / E_{\text {acc }}$ & $\ldots$ & $\ldots$ & 0.60 & 0.79 \\
\hline \hline
\end{tabular}

Using VORPAL, figures of merit for the accelerating mode in each cavity type were calculated and compared for a periodic single cell with iris radius $a=3.15 \mathrm{~mm}$, iris thickness $d=1.67 \mathrm{~mm}$, and phase advance $\phi=2 \pi / 3$ and are summarized in Table I. The quality factors quantify power losses due to various physical mechanisms: (i) $Q_{\mathrm{rad}}$ : losses due to radiation leaking through the truncated $\mathrm{PhC}$ structures (confinement of the accelerating mode is not perfect). Applies to hybrid $\mathrm{PhC}$ cavities only. (ii) $Q_{\text {metal }}$ : losses due to rf heating of copper surfaces. (iii) $Q_{\text {diel }}$ : losses due to $\mathrm{rf}$ heating of sapphire rods. Applies to hybrid $\mathrm{PhC}$ cavities only. We use a conservative value for the sapphire loss tangent: $10^{-4}$.

The shunt impedance per unit length is

$$
r_{\text {shunt }}=\frac{V^{2}}{P L}
$$

where $V$ is the accelerating voltage across a single cell, $P$ is the total power loss per cell, and $L$ is the length of a single cell ( $L=8.33 \mathrm{~mm}$ based on the synchronicity condition). The accelerating gradient is simply $E_{\text {acc }}=V / L$. The loss factor per unit length is $k=V^{2} / 4 U L$ where $U$ is the accelerating mode stored energy. The group velocity of the accelerating mode is $v_{g}$, obtained from two simulations at different phase advances.

\section{Surface fields}

Figures 17 and 18 show the absolute values of $E_{z}$ and $\mathbf{B}_{\perp}$ on the $z$ midplane of the relevant periodic single cell cavities. The $z$ dependences of the fields are very similar amongst the different cavity types, as expected based on the common iris geometry in each structure. The maximum surface electric field occurs on the iris in all cases, explaining the uniformity of $E_{\text {metal,surf,max }} / E_{\text {acc }}$ across all cavity types. The maximum surface magnetic field occurs on the innermost radial walls of the CLIC cavity; the elliptic curvature of this feature was carefully chosen to minimize $c B_{\text {surf }} / E_{\text {acc }}$.

In the hybrid $\mathrm{PhC}$ cavities, $B_{\text {metal,surf,max }}$ occurs where the innermost rods abut the conducting endplates. Since the maximum occurs at the interface between dielectric and conductor, the method used to hold the rods in place will require careful consideration. For example, brazing material could be more prone to breakdown or suffer greater heating losses than copper. The experiment in Ref. [4] successfully secured the rods simply by endplate pressure. On the other hand, covering the region of conductor suffering the maximum magnetic field with dielectric could suppress the breakdown mechanism. Of course, only experiment will tell.

\section{Power losses/accelerating efficiency}

The reduced copper surface area in the hybrid $\mathrm{PhC}$ cavities lowers copper heating losses compared to CLIC and the pillbox; however (unlike the all-copper cavities), dielectric heating and radiative losses play a role. The shunt impedance is a strong indicator of accelerating efficiency,

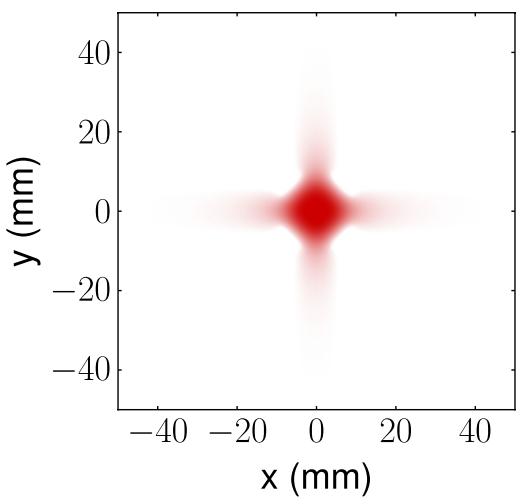

(a) CLIC

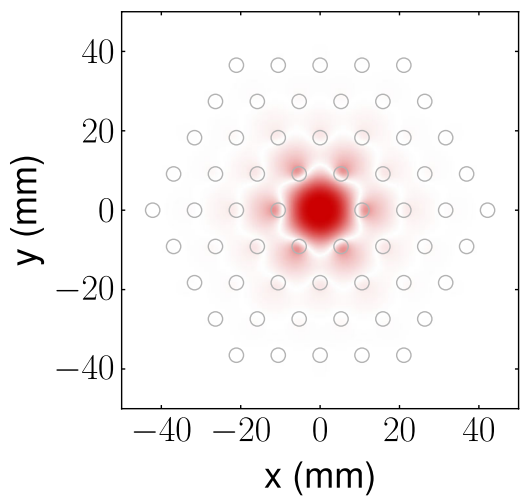

(b) Tri-4-Sapphire

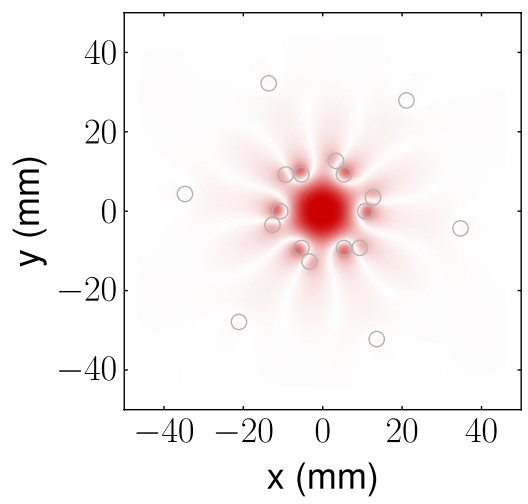

(c) Opt-18-Sapphire

FIG. 17. Absolute value of $E_{z}$ on the midplane (in $z$ ) of periodic single cell cavities. 


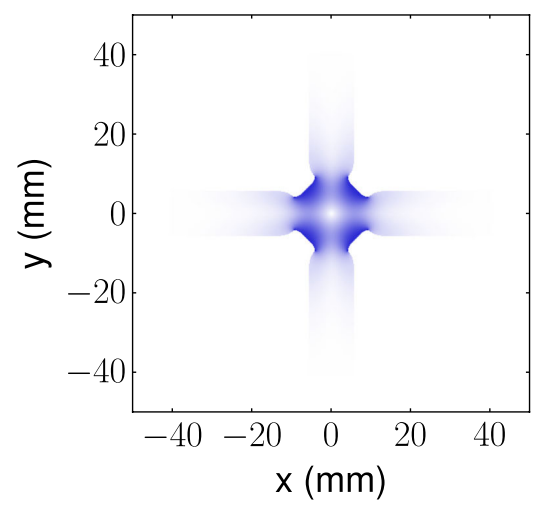

(a) CLIC

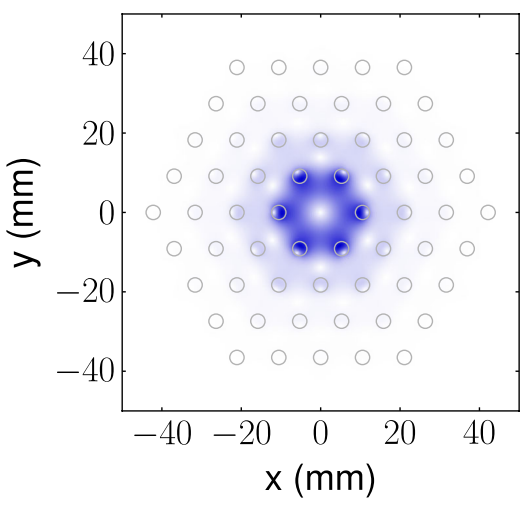

(b) Tri-4-Sapphire

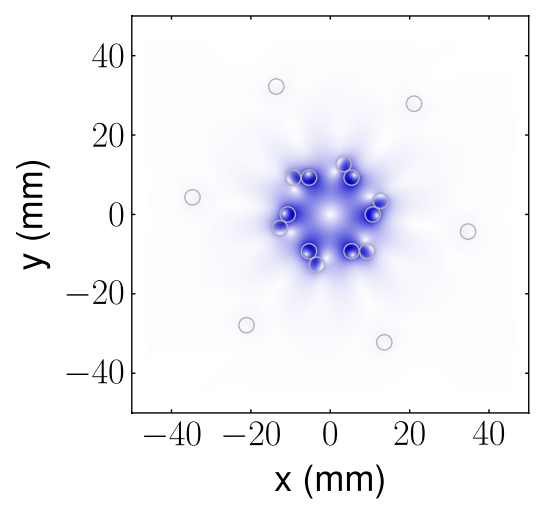

(c) Opt-18-Sapphire

FIG. 18. Absolute value of $\mathbf{B}_{\perp}$ on the midplane (in $z$ ) of periodic single cell cavities.

and is higher in the CLIC cavity. However, shunt impedances in the hybrid $\mathrm{PhC}$ cavities could be increased by reducing radiative losses (e.g., by adding more layers of rods to the Tri-4-Sapphire cavity, or performing optimizations in 3D on the Opt-18-Sapphire cavity as discussed below).

In the optimized cavity, $Q_{\text {rad }}$ is very sensitive to structural perturbations (cf. Ref. [10]). We find that the beam tubes in the 3D Opt-18-Sapphire cavity drop $Q_{\text {rad }}$ by nearly an order of magnitude relative to the $2 \mathrm{D}$ value reported in Ref. [10] (from 25,000 in $2 \mathrm{D}$ to 3,800 in 3D with beam tubes). It remains to be seen whether further optimization in 3D can regain the original $2 \mathrm{D} Q_{\text {rad }}$. The $Q_{\text {rad }}$ of the Tri-4-Sapphire cavity is the same in 2D, indicating some robustness of the confinement to structural perturbations, likely due to the band gap trapping mechanism.

[1] E. R. Colby, AIP Conf. Proc. 647, 39 (2002).

[2] D. R. Smith, D. Li, D. C. Vier, N. Kroll, and S. Schultz, AIP Conf. Proc. 398, 518 (1997).

[3] N. Kroll, D. R. Smith, and S. Schultz, AIP Conf. Proc. 279, 197 (1993).

[4] M. R. Masullo, A. Andreone, E. Di Gennaro, S. Albanese, F. Francomacaro, M. Panniello, V. G. Vaccaro, and G. Lamura, Microwave Opt. Technol. Lett. 48, 2486 (2006).

[5] E. Gennaro, C. Zannini, S. Savo, A. Andreone, M. Masullo, G. Castaldi, I. Gallina, and V. Galdi, New J. Phys. 11, 113022 (2009).

[6] E. Di Gennaro, S. Savo, A. Andreone, V. Galdi, G. Castaldi, V. Pierro, and M. Masullo, Appl. Phys. Lett. 93, 164102 (2008).

[7] M. Shapiro, E. Smirnova, C. Chen, and R. Temkin, in Proceedings of the 2003 Particle Accelerator Conference, Portland, OR (IEEE, New York, 2004), Vol. 2, pp. 1255-1257.

[8] E. I. Smirnova, A. S. Kesar, I. Mastovsky, M. A. Shapiro, and R. J. Temkin, Phys. Rev. Lett. 95, 074801 (2005).
[9] R. A. Marsh, M. A. Shapiro, R. J. Temkin, V. A. Dolgashev, L. L. Laurent, J. R. Lewandowski, A. D. Yeremian, and S. G. Tantawi, Phys. Rev. ST Accel. Beams 14, 021301 (2011).

[10] C. Bauer, G. Werner, and J. Cary, J. Appl. Phys. 104, 053107 (2008).

[11] B. Cowan, Phys. Rev. ST Accel. Beams 11, 011301 (2008).

[12] X. Lin, Phys. Rev. ST Accel. Beams 4, 051301 (2001).

[13] M. Thompson, H. Badakov, A. Cook, J. Rosenzweig, R. Tikhoplav, G. Travish, I. Blumenfeld, M. Hogan, R. Ischebeck, N. Kirby et al., Phys. Rev. Lett. 100, 214801 (2008).

[14] N. Bloembergen, IEEE J. Quantum Electron. 10, 375 (1974).

[15] D. Du, X. Liu, G. Korn, J. Squier, and G. Mourou, Appl. Phys. Lett. 64, 3071 (1994).

[16] J. G. Power, W. Gai, S. H. Gold, A. K. Kinkead, R. Konecny, C. Jing, W. Liu, and Z. Yusof, Phys. Rev. Lett. 92, 164801 (2004).

[17] C. Jing, W. Gai, J. Power, R. Konecny, W. Liu, S. Gold, A. Kinkead, S. Tantawi, V. Dolgashev, and A. Kanareykin, IEEE Trans. Plasma Sci. 38, 1354 (2010).

[18] G. R. Werner, C. A. Bauer, and J. R. Cary, Phys. Rev. ST Accel. Beams 12, 071301 (2009).

[19] R. A. Marsh, Ph.D. thesis, Massachusetts Institute of Technology, 2009.

[20] C. Jing, F. Gao, S. Antipov, Z. Yusof, M. Conde, J. G. Power, P. Xu, S. Zheng, H. Chen, C. Tang et al., Phys. Rev. ST Accel. Beams 12, 121302 (2009).

[21] I. Wilson and W. Wuensch, arXiv:physics/0008101.

[22] D. Trines, Linear Accelerator. Proceedings, 22nd International Conference, Linac 2004, Luebeck, Germany, 2004.

[23] H. Braun, L. Rinolfi, S. Weisz, A. Ferrari, R. Tomás, G. Geschonke, F. Tecker et al., CLIC 2008 parameters. Report No. CERN-OPEN-2008-021, 2008.

[24] A. Grudiev (private communication).

[25] A. Grudiev, Possible Improvement of the CLIC Accelerating Structure: From CLIC-G to CLIC-K (SLAC National Accelerator Laboratory, USA, 2009).

[26] N. Kroll and X. Lin, in Proceedings of the 15th Particle Accelerator Conference, Washington, DC, 1993 (IEEE, New York, 1993), pp. 3453-3455. 
[27] X. Lin and N. Kroll, in Proceedings of the Particle Accelerator Conference, Dallas, TX, 1995 (IEEE, New York, 1995), Vol. 3, pp. 1809-1811.

[28] S. G. Johnson and J. D. Joannopoulos, Opt. Express 8, 173 (2001).

[29] D. Li, N. Kroll, D. Smith, and S. Schultz, AIP Conf. Proc. 398, 528 (1997).

[30] P. B. Wilson, SLAC Technical Report No. SLAC-PUB4547, 1989.

[31] C. Nieter and J. R. Cary, J. Comput. Phys. 196, 448 (2004).
[32] O. Napoly, Y. Ho Chin, and B. Zotter, Nucl. Instrum. Methods Phys. Res., Sect. A 334, 255 (1993).

[33] T. Weiland, Nucl. Instrum. Methods Phys. Res. 216, 31 (1983).

[34] J. D. Joannopoulos, S. G. Johnson, J. N. Winn, and R. D. Meade, Photonic Crystals: Molding the Flow of Light (Princeton University Press, Princeton, NJ, 2011).

[35] M. Luong, I. Wilson, and W. Wuensch, in Proceedings of the 18th Particle Accelerator Conference, New York, 1999 (IEEE, New York, 1999), Vol. 2, pp. 821-823. 